\title{
SUB-SELF-SIMILAR SETS
}

\author{
K. J. FALCONER
}

\begin{abstract}
A compact set $E \subseteq \mathbf{R}^{n}$ is called sub-self-similar if $E \subseteq \bigcup_{i=1}^{m} S_{i}(E)$, where the $S_{i}$ are similarity transfunctions. We consider various examples and constructions of such sets and obtain formulae for their Hausdorff and box dimensions, generalising those for self-similar sets.
\end{abstract}

\section{INTRODUCTION}

A wide range of fractals are self-similar in the sense that they are made up of arbitrarily small similar copies of themselves. Self-similar sets were presented in a unified way by Hutchinson [8], and an enormous literature has developed concerning the representation of self-similar sets and the calculation of their dimensions and measures, see for example $[1,2,7,12]$.

Recall that $S: \mathbf{R}^{n} \rightarrow \mathbf{R}^{n}$ is a similarity with ratio $c$ if

$$
|S(x)-S(y)|=c|x-y| \quad\left(x, y \in \mathbf{R}^{n}\right) ;
$$

if $c<1$ we say that $S$ is contracting. We assume throughout that the similarities considered are nondegenerate, i.e. $c>0$. A fundamental result, proved by Hutchinson using the contraction mapping theorem, is that given $m \geq 2$ and contracting similarities $S_{i}: \mathbf{R}^{n} \rightarrow \mathbf{R}^{n}(i=1,2, \ldots, m)$ there exists a unique nonempty compact set $E \subseteq \mathbf{R}^{n}$ satisfying

$$
E=\bigcup_{i=1}^{m} S_{i}(E) .
$$

This set $E$ is called self-similar, or self-similar for $\left\{S_{1}, \ldots, S_{m}\right\}$ if the similarity transfunctions need to be emphasised. (Note that the terminology varies between authors.) Formulae for the Hausdorff and other dimensions of selfsimilar sets in terms of the similarity ratios have been given under certain conditions; usually the components $S_{i}(E)(i=1, \ldots, m)$ of $E$ are required to satisfy some separation condition.

In this paper we introduce a generalisation of self-similar sets by relaxing equality in (1.1) to inclusion. With $m \geq 2$ and $S_{i}: \mathbf{R}^{n} \rightarrow \mathbf{R}^{n}$ contacting similarities $(i=1,2, \ldots, m)$ we term a nonempty compact set $E \subseteq \mathbf{R}^{n}$ sub-

Received by the editors March 10, 1994; originally communicated to the Proceedings of the AMS by Palle E. T. Jorgenson.

1991 Mathematics Subject Classification. Primary 28A75. 
self-similar (s.s.s.) for $\left\{S_{1}, \ldots, S_{m}\right\}$ if

$$
E \subseteq \bigcup_{i=1}^{m} S_{i}(E) .
$$

These sets include many interesting examples, such as boundaries of self-similar sets. Despite the level of generality, it is possible to find formulae for the Hausdorff and box-counting dimensions of s.s.s. sets valid under reasonable conditions. In Section 3 we obtain such formulae which extend the (by now) standard result for self-similar sets.

\section{EXAMPLES OF SUB-SELF-SIMILAR SETS}

In this section we demonstrate the scope of the definition by giving a variety of examples and constructions of s.s.s. sets.

(a) Self-similar sets. Trivially the self-similar set $E$ that satisfies (1.1) is s.s.s. for $\left\{S_{1}, \ldots, S_{m}\right\}$.

(b) Recursively self-similar sets. These are a generalisation of self-similar sets, see $[3,10]$. Let $m \geq 2$ be an integer and let $G$ be a subset of $\{(i, j): 1 \leq i, j \leq$ $m$ \} such that the directed graph defined by $G$ is transitive. For $(i, j) \in G$ let $S_{i, j}: \mathbf{R}^{n} \rightarrow \mathbf{R}^{n}$ be a contacting similarity. It may be shown [3, Theorem 4.3.5] that there exists a unique family of nonempty compact sets $E_{1}, \ldots, E_{m}$ such that

$$
E_{i}=\bigcup_{j=1}^{m} S_{i, j}\left(E_{j}\right)
$$

for $i=1,2, \ldots, m$. The sets $\left\{E_{1}, \ldots, E_{m}\right\}$ are known as a recursively selfsimilar family of sets. It is easy to see that $\bigcup_{i=1}^{m} E_{i}$ is s.s.s. for $\left\{S_{i, j}:(i, j) \in\right.$ $G\}$. By adding translations to the $S_{i, j}$ we can translate the $\left\{E_{i}: 1 \leq i \leq m\right\}$ to disjoint sets, enabling them to be studied separately.

(c) Unions of s.s.s. sets. If $E$ is s.s.s. for $\left\{S_{1}, \ldots, S_{m}\right\}$ and $E^{\prime}$ is s.s.s. for $\left\{S_{1}^{\prime}, \ldots, S_{m^{\prime}}^{\prime}\right\}$, then $E \cup E^{\prime}$ is s.s.s. for $\left\{S_{1}, \ldots, S_{m}, S_{i}^{\prime}, \ldots, S_{m^{\prime}}^{\prime}\right\}$.

(d) Augmenting s.s.s. sets. Let $E \subseteq \mathbf{R}^{n}$ be s.s.s. for $\left\{S_{1}, \ldots, S_{m}\right\}$, let $F$ be a compact subset of $E$, and let $S$ be a contracting similarity. Then $E \cup S(F)$ is s.s.s. for $\left\{S_{1}, \ldots, S_{m}, S\right\}$.

(e) Reduction of s.s.s. sets. The following construction enables us to extract s.s.s. subsets of s.s.s. sets with particular local properties.

Let $E$ be s.s.s. with respect to $\left\{S_{1}, \ldots, S_{m}\right\}$. Let $P(A, x)$ be a property that depends on a set $A$ and point $x$ in $\mathbf{R}^{n}$. Assume that

(i) if $P(A, x)$ holds, then $x \in \bar{A}$;

(ii) if $A \subseteq \bigcup_{i=1}^{m} A_{i}$ and $P(A, x)$ holds, then $P\left(A_{i}, x\right)$ holds for some $i$ $(1 \leq i \leq m)$;

(iii) if $P(A, x)$ holds, then $P(S(A), S(x))$ holds for all similarities $S$.

Define

$$
E^{\prime}=\{x \in E ; P(E, x) \text { holds }\} \text {. }
$$

If $E^{\prime}$ is compact, then it is also s.s.s. with respect to $\left\{S_{1}, \ldots, S_{m}\right\}$. 
To check this, if $x \in E^{\prime}$, then $P(E, x)$ holds, so applying (ii) to the inclusion $E \subseteq \bigcup_{i=1}^{m} S_{i}(E)$ gives that $P\left(S_{i}(E), x\right)$ holds for some $i$. By (iii) $P\left(E, S_{i}^{-1}(x)\right)$ holds, so by (i) $S_{i}^{-1}(x) \in \bar{E}=E$; thus $S_{i}^{-1}(x) \in E^{\prime}$, as required.

There are many relevant properties $P$ that satisfy (i)-(iii) above. For examples, fixing $\alpha>0$ and taking $P(A, x)$ to be " $\operatorname{dim}_{H}\left(A \cap N_{x}\right) \geq \alpha$ for all neighbourhoods $N_{x}$ of $x$ " where $\operatorname{dim}_{H}$ is Hausdorff dimension (see Section 3 for a definition), (i)-(iii) are satisfied, so for any s.s.s. set $E$,

$$
E^{\prime}=\left\{x \in E: \operatorname{dim}\left(E \cap N_{x}\right) \geq \alpha \text { for all neighbourhoods } N_{x} \text { of } x\right\}
$$

is S.S.S.

(f) Boundaries. Let $E$ be a self-similar set satisfying (1.1) and let $\partial E$ be the topological boundary of $E$, i.e. any open set intersecting $\partial E$ contains points both of $E$ and its complement. Then $\partial E$ is s.s.s. for $\left\{S_{1}, \ldots, S_{m}\right\}$, for, if $x \in$ $\partial E$, then $x \in E$, so $x \in S_{j}(E)$ for some $j$. However, every neighbourhood $N_{x}$ of $x$ contains points outside $E=\bigcup_{i=1}^{m} S_{i}(E)$, so in particular any such neighbourhood contains points outside $S_{j}(E)$. Thus $x \in \partial S_{j}(E)=S_{j}(\partial E)$, giving that $\partial E$ is s.s.s.

Various authors have represented clouds as self-similar sets with positive volume; thus their (fractal) boundaries are s.s.s.

(g) Sets indexed by sequences. Much of the theory that has been developed for self-similar sets depends on coding the points of the set by sequences of the form $\left\{\left(i_{1}, i_{2}, \ldots\right): 1 \leq i_{j} \leq m\right\}$ or equivalently regarding the set as a continuous image of $\{1,2, \ldots, m\}^{\mathbf{N}}$ with the product topology, see, for example [7, 8]. A similar approach may be adopted here: s.s.s. sets may be defined in terms of sequences, and we will see that every s.s.s. set has a sequence representation.

Fix $m \geq 2$, let $S_{1}, \ldots, S_{m}$ be contracting similarities on $\mathbf{R}^{n}$, and let $c_{i}$ be the contraction ratio of $S_{i}(i=1, \ldots, m)$. Let $J=\left\{\left(i_{1}, i_{2}, \ldots,\right): 1 \leq\right.$ $\left.i_{j} \leq m\right\}$ be the set of infinite sequences of terms chosen from $\{1,2, \ldots, m\}$. Define a metric $d$ on $J$ by

$$
d\left(\left(i_{1}, \ldots, i_{k}, i_{k+1}, \ldots\right),\left(i_{1}, \ldots, i_{k}, j_{k+1}, \ldots\right)\right)=c_{i_{1}} \cdots c_{i_{k}}
$$

where $i_{k+1} \neq j_{k+1}$ (with the obvious convention of taking $d$ to be 0 if the sequences are equal and 1 if they differ the first term).

Let $B$ be any closed ball in $\mathbf{R}^{n}$ large enough to ensure that $S_{i}(B) \subseteq B$ for all $i(i=1,2, \ldots, m)$. We note that if $\mathbf{i}=\left(i_{1}, i_{2}, \ldots\right) \in J$ the sequence of balls $S_{i_{i}} \circ \cdots \circ S_{i_{k}}(B)$ decreases with $k$ and has intersection a single point, and we write

$$
a(\mathbf{i})=\bigcap_{k=1}^{\infty} S_{i_{1}} \circ \cdots \circ S_{i_{k}}(B) .
$$

Since the $S_{i}$ are contracting it is easy to see that $a(\mathbf{i})$ is independent of the ball $B$ chosen. Moreover, if $z$ is any point of $\mathbf{R}^{n}$ we have

$$
a(\mathbf{i})=\lim _{k \rightarrow \infty} S_{i_{1}} \circ \cdots \circ S_{i_{k}}(z) .
$$

It follows easily from (2.1) that the mapping $A: J \rightarrow \mathbf{R}^{n}$ is Lipschitz and thus continuous, though it need not be injective. The set $A=\bigcup_{\mathbf{i} \in J} a(\mathbf{i})$ is precisely 
the self-similar set satisfying (1.1). It may be shown that if $a$, regarded as a mapping $J \rightarrow A$, is injective (and thus bijective), then it is bi-Lipschitz, i.e. its inverse is also Lipschitz.

Now let $K$ be a compact subset of $J$ that is closed under the left shift, i.e. $\left(i_{2}, i_{3}, \ldots\right) \in K$ whenever $\left(i_{1}, i_{2}, \ldots\right) \in K$. We show that $a(K)$ is s.s.s together with the converse property that any s.s.s. set may be characterised in this way.

Proposition 2.1. Let $S_{1}, \ldots, S_{m}$ be contracting similarities on $\mathbf{R}^{n}$. Then $E$ is compact and sub-self-similar for $\left\{S_{1}, \ldots, S_{m}\right\}$ if and only if $E=a(K)$ for some compact set $K \subseteq J$ satisfying the condition

$$
\left(i_{1}, i_{2}, \ldots\right) \in K \Rightarrow\left(i_{2}, i_{3}, \ldots\right) \in K \text {. }
$$

Proof. Suppose that $K$ is a compact subset of $J$ satisfying (2.4), and consider the set $a(K)$, where $a$ is given by (2.3). If $x \in a(K)$, then for all $z \in \mathbf{R}^{n}$

$$
x=a(\mathbf{i})=\lim _{k \rightarrow \infty} S_{i_{1}} \circ S_{i_{2}} \circ \cdots \circ S_{i_{k}}(z)
$$

for some $\mathbf{i}=\left(i_{1}, i_{2}, \ldots\right) \in K$. By $(2.4) \mathbf{i}^{\prime}=\left(i_{2}, i_{3}, \ldots\right) \in K$, so

$$
x=S_{i_{1}}\left(a\left(\mathbf{i}^{\prime}\right)\right) \in S_{i_{1}}(a(K)) .
$$

Hence $a(K)$ satisfies (1.2).

Conversely, suppose that $E$ is a compact subset of $\mathbf{R}^{n}$ satisfying (1.2). Define $K=\left\{\left(i_{1}, i_{2}, \ldots\right): a\left(i_{k}, i_{k+1}, \ldots\right) \in E\right.$ for all $\left.k \in \mathbf{Z}^{+}\right\}$. Condition (2.4) is satisfied trivially. Since $E$ is closed and $a: J \rightarrow \mathbf{R}^{n}$ is continuous, $a^{-1}(E)$ is closed and so $K=\bigcap_{k=1}^{\infty}\left\{\left(i_{1}, i_{2}, \ldots\right):\left(i_{k}, i_{k+1}, \ldots\right) \in a^{-1}(E)\right\}$ is closed and thus compact. Clearly, $a(K) \subseteq E$. If $x_{0} \in E$, then by (1.2) $x_{0}=S_{i_{1}}\left(x_{1}\right)$ for some $x_{1} \in E$ and $1 \leq i_{1} \leq m$; similarly $x_{1}=S_{i_{2}}\left(x_{2}\right)$ for some $x_{2} \in E$ and $1 \leq i_{2} \leq m$, and so on. It follows that for $k \in \mathbf{Z}^{+}$we have $a\left(i_{k}, i_{k+1}, \ldots\right)=\bigcap_{j=k}^{\infty} S_{i_{k}} \circ \cdots \circ S_{i_{j}}(B)=x_{k-1} \in E$, where $B$ is a closed ball with $B \supseteq E$ and $S_{i}(B) \subseteq B$ for all $i=1,2, \ldots, m$, so in particular $x_{0} \in a(K)$, giving $E \subseteq a(K)$.

It has been pointed out that a proof of Proposition 2.1 may be found in Bandt [2].

One way of constructing sets satisfying (2.4) is, essentially, as the closure of an orbit under the left shift. Thus if $\left(i_{1}, i_{2}, \ldots\right) \in J$ is given, the set $K=\overline{\left\{\left(i_{k}, i_{k+1}, \ldots\right): k \in \mathbf{Z}^{+}\right\}}$satisfies $(2.4)$ and so leads to s.s.s. sets.

\section{DimENSIONS OF SUB-SELF-SIMILAR SETS}

In this section we obtain some results on the Hausdorff dimensions of s.s.s. sets, generalising those for self-similar sets, as well as conditions for the Hausdorff measure at the critical dimension to be positive or finite, see $[1,6,7$ (Chapter 9), 8, 12].

Recall that for $0 \leq s \leq n$ the $s$-dimensional Hausdorff (outer) measure of a set $E \subseteq \mathbf{R}^{n}$ is given by

$$
\begin{gathered}
H^{s}(E)=\lim _{\delta \rightarrow 0} H_{\delta}^{s}(E), \\
H_{\delta}^{s}(E)=\inf \left\{\sum_{i=1}^{\infty}\left|U_{i}\right|^{s}: E \subseteq \bigcup_{i=1}^{\infty} U_{i} \text { and }\left|U_{i}\right| \leq \delta\right\},
\end{gathered}
$$


where $|U|$ is the diameter of the set $U$. The Hausdorff dimension of $E$, denoted by $\operatorname{dim}_{H} E$, is the number such that $H^{s}(E)=\infty$ if $0 \leq s<\operatorname{dim}_{H} E$ and $H^{s}(E)=0$ if $\operatorname{dim}_{H} E<s \leq n$. Writing $N_{\delta}(E)$ for the minimum number of sets of diameter $\delta$ which can cover $E$, we define the lower and upper boxcounting dimensions of a nonempty bounded set $E$ by

$$
\underline{\operatorname{dim}}_{B} E=\underline{\lim }_{\delta \rightarrow 0} \log N_{\delta}(E) /-\log \delta
$$

and

$$
\overline{\operatorname{dim}}_{B} E=\varlimsup_{\delta \rightarrow 0} \log N_{\delta}(E) /-\log \delta
$$

respectively. If these two numbers are equal, we write $\operatorname{dim}_{B} E$ for the common value.

These dimensions satisfy

$$
\operatorname{dim}_{H} E \leq \underline{\operatorname{dim}}_{B} E \leq \overline{\operatorname{dim}}_{B} E
$$

for any set $E$. Virtually all the commonly used definitions of dimension take a value between $\operatorname{dim}_{H}$ and $\overline{\operatorname{dim}}_{B}$; thus it is extremely useful to have conditions on a set $E$ that guarantee that these values are equal. In this section we show that if the family of similarity transformations underlying an s.s.s. set satisfies the open set condition, then equality holds in (3.1), and we obtain an expression for this common value.

Recall that the family of similarities $\left\{S_{1}, \ldots, S_{m}\right\}$ satisfies the open set condition if there is a bounded nonempty open set $V$ such that

$$
\bigcup_{i=1}^{m} S_{i}(V) \subseteq V
$$

with this union disjoint. The self-similar set $E$ defined for these similarities by (1.1) is contained in the closure $\bar{V}$ of any such $V$. Any s.s.s. set for $\left\{S_{1}, \ldots, S_{m}\right\}$ is necessarily a subset of $E$ and so of $\bar{V}$.

We use the sequence terminology of Section $1(\mathrm{~g})$ in our dimension calculations, noting that Proposition 2.1 allows this for any s.s.s. set. Let $E$ be s.s.s with respect to $\left\{S_{1}, \ldots, S_{m}\right\}$, and recall that

$$
K=\left\{\left(i_{1}, i_{2}, \ldots\right): a\left(i_{k}, i_{k+1}, \ldots\right) \in E \text { for all } k \in \mathbf{Z}^{+}\right\} \subseteq J
$$

with $a: J \rightarrow E$ as in (2.2). For $k \in \mathbf{Z}^{+}$define $J_{k}=\{\mathbf{i} \mid k: \mathbf{i} \in J\}$ and $K_{k}=$ $\{\mathbf{i} \mid k: \mathbf{i} \in K\}$ where $\mathbf{i} \mid k$ denotes the $k$-term sequence obtained by curtailing i after $k$ terms. The following observation is crucial to the submultiplicity argument that follows. Let $\mathbf{i} \in J_{k}$ and $\mathbf{j} \in J_{l}$ and write ij for the sequence in $J_{k+l}$ obtained by concatenation. Then

$$
\mathbf{i j} \in K_{k+l} \text { implies that both } \mathbf{i} \in K_{k} \text { and } \mathbf{j} \in K_{l}
$$

(that $\mathbf{i} \in K_{k}$ is immediate from the definition of $K_{k}$, and that $\mathbf{j} \in K_{l}$ follows since $K$ satisfies (2.4)).

Let $c_{i}$ be the ratio of the similarity $S_{i}$ (recall $0<c_{i}<1$ ) for $i=1, \ldots, m$ and write $c_{\mathbf{i}}=c_{i_{1}} c_{i_{2}} \cdots c_{i_{k}}$, where $\mathbf{i}=\left(i_{1}, \ldots, i_{k}\right)$, for the ratio of the composition $S_{i_{1}} \circ \cdots \circ S_{i_{k}}$. Clearly $c_{\mathbf{i j}}=c_{\mathbf{i}} c_{\mathbf{j}}$, so (3.3) implies that, for $s \geq 0$,

$$
\sum_{\mathbf{i} \in K_{k+l}} c_{\mathbf{i}}^{s} \leq \sum_{\mathbf{i} \in K_{k}} \sum_{\mathbf{j} \in K_{l}} c_{\mathbf{i j}}^{s}=\left(\sum_{\mathbf{i} \in K_{k}} c_{\mathbf{i}}^{s}\right)\left(\sum_{\mathbf{i} \in K_{l}} c_{\mathbf{i}}^{s}\right) .
$$


Hence $\left(\sum_{\mathbf{i} \in K_{k}} c_{\mathbf{i}}^{S}\right)$ is a submultiplicative sequence, so by the standard properties of such sequences, we have that the limit

$$
\tau(s) \equiv \lim _{k \rightarrow \infty}\left(\sum_{\mathbf{i} \in K_{k}} c_{\mathbf{i}}^{s}\right)^{1 / k}
$$

exists with $0 \leq \tau(s)<\infty$ and $\sum_{\mathbf{i} \in K_{k}} c_{\mathbf{i}}^{s} \geq \tau(s)^{k}$ for all $k=1,2, \ldots$.

We shall show that, given the open set condition, the Hausdorff and box dimensions of $E$ equal the unique nonnegative $s$ satisfying $\tau(s)=1$; we first obtain alternative characterisations of this number. The basic approach is similar to that of Hutchinson [8]; that it can be adapted to this situation depends crucially on (3.3) and the consequent submultiplicity condition (3.4). One complication is that s.s.s. sets need not have finite $s$-dimensional Hausdorff measure and this requires us to invoke powerful results on the existence of subsets of finite measure; a related procedure was used in calculating dimensions of self-affine sets [5].

It is convenient to define auxillary measures of Hausdorff type on subsets of $K$ which we then transfer to $E$. Let $s \geq 0$. For $k=1,2, \ldots$ and $A \subseteq J$ let

$$
M_{k}^{s}(A)=\inf \left\{\sum_{\mathbf{i}} c_{\mathbf{i}}^{s}: A \subseteq \bigcup_{\mathbf{i}} I_{\mathbf{i}},|\mathbf{i}| \geq k\right\}
$$

where we define the cylinders $I_{\mathbf{i}}=\{\mathbf{i j}: \mathbf{j} \in J\}$ for $\mathbf{i} \in J_{k}$. For each $k$ this defines an outer measure on subsets of $J$ and we obtain a Borel measure of Hausdorff type by letting

$$
M^{s}(A)=\lim _{k \rightarrow \infty} M_{k}^{s}(A)
$$

(see, for example, Rogers [11] for a full treatment of this method of constructing such measures).

We will require the following form of Frostman's lemma which enables us to reduce infinite measures to finite ones.

Proposition 3.1. Suppose that $0<M^{s}(A) \leq \infty$ for some $s \geq 0$ and $A$ is a Borel subset of $J$. Then there exists a compact set $A_{0} \subseteq A$ and a constant $b>0$ such that $0<M^{s}\left(A_{0}\right)<\infty$ and

$$
M^{s}\left(A_{0} \cap I_{\mathbf{i}}\right) \leq b c_{\mathbf{i}}^{s}
$$

for all $\mathbf{i} \in J$.

Proof. This is essentially Rogers [11, Theorem 54]; inequality (3.7) may be gleaned from the proof given there. Alternatively, it follows as in Falconer [4, Theorem 5.4] or Mattila [9 Theorem 8.8].

Proposition 3.2. Let $K$ be an s.s.s. set. The following numbers exist and are equal:

(a) the unique $s \geq 0$ such that $\tau(s)=1$,

(b) $\inf \left\{s \geq 0: M^{s}(K)=0\right\}=\sup \left\{s \geq 0: M^{s}(K)=\infty\right\}$,

(c)

$$
\inf \left\{s \geq 0: \sum_{k=1}^{\infty} \sum_{\mathbf{i} \in K_{k}} c_{\mathbf{i}}^{s}<\infty\right\}=\sup \left\{s \geq 0: \sum_{k=1}^{\infty} \sum_{\mathbf{i} \in K_{k}} c_{\mathbf{i}}^{s}=\infty\right\} .
$$


(In (b) and (c) we make the obvious convention that the supremum of the empty set is 0.) Moreover, for this value of $s$,

$$
M^{s}(K) \geq 1 .
$$

Proof. To justify (a), writing $c_{-}=\min _{1 \leq i \leq m} c_{i}$ and $c_{+}=\max _{1 \leq i \leq m} c_{i}$, we have for $h>0$

$$
c_{-}^{k h} \leq\left(\sum_{\mathbf{i} \in K_{k}} c_{\mathbf{i}}^{s+h}\right) /\left(\sum_{\mathbf{i} \in K_{k}} c_{\mathbf{i}}^{s}\right) \leq c_{+}^{k h} .
$$

Using (3.5) it follows that $c_{-}^{h} \leq \tau(s+h) / \tau(s) \leq c_{+}^{h}$ for $s \geq 0$ and $h>0$, so $\tau$ is continuous and strictly decreasing. Moreover, $\tau(0) \geq 1$ and $\tau(s) \leq 1$ if $s \geq-\log m / \log c_{+}$. Hence there is a unique $s \geq 0$ satisfying $\tau(s)=1$.

The equality of the inf and sup in (b) is a standard feature of measures of Hausdorff type, and the equality of the inf and sup in (c) is obvious.

To see that $(\mathrm{a})=(\mathrm{c})$ we observe that $\sum_{k=1}^{\infty} \sum_{\mathbf{i} \in K_{k}} c_{\mathbf{i}}^{s}$ converges if $\tau(s)<1$ and diverges if $\tau(s)>1$.

In calculating $M^{s}(K)$ we need only use covering cylinders $I_{\mathbf{i}}$ with $\mathbf{i} \in$ $\bigcup_{k=0}^{\infty} K_{k}$ (other cylinders are disjoint from $K$ ), so if $\sum_{k=1}^{\infty} \sum_{\mathbf{i} \in K_{k}}^{\infty} c_{\mathbf{i}}^{s}<\infty$, then $M_{k}^{s}(K) \leq \sum_{j=k}^{\infty} \sum_{\mathbf{i} \in K_{j}} c_{\mathbf{i}}^{s} \rightarrow 0$ as $k \rightarrow \infty$. Thus (b) $\leq$ (c) .

Finally, we show that (a) $\leq(\mathrm{b})$ and also (3.8). Suppose that $M^{s}(K)<1$ for some $s>0$. Then there is a covering of $K$ by cylinders $\bigcup_{i \in Q} I_{i}$ with $\sum_{\mathbf{i} \in Q} c_{\mathbf{i}}^{S}<1$ and thus with

$$
\sum_{\mathbf{i} \in Q} c_{\mathbf{i}}^{t}<1
$$

for some $t$ with $0<t<s$; using the compactness of $K$ we may take $Q$ to be finite. Write $q=\max \{|\mathbf{i}|: \mathbf{i} \in Q\}$. We define further families of indices $Q_{k}(k \geq q)$ by

$$
Q_{k}=\left\{\mathbf{i}_{1} \mathbf{i}_{2} \cdots \mathbf{i}_{p}: \mathbf{i}_{j} \in Q,\left|\mathbf{i}_{1} \mathbf{i}_{2} \cdots \mathbf{i}_{p}\right| \geq k \text { and }\left|\mathbf{i}_{1} \mathbf{i}_{2} \cdots \mathbf{i}_{p-1}\right|<k\right\}
$$

where $|\mathbf{i}|$ denotes the number of terms in the sequence $\mathbf{i}$. It follows using (3.3) that $K \cap I_{\mathbf{i}} \subseteq \bigcup_{\mathbf{j} \in Q}\left(K \cap I_{\mathbf{i j}}\right)$ for $\mathbf{i} \in K_{k}$ and all $k$. Thus if $\mathbf{i} \in K_{k}$, then $\mathbf{i j} \in Q_{k}$ for some $\mathbf{j}$ with $|\mathbf{j}| \leq q$. Hence, we have that for each $k$

$$
\sum_{\mathbf{i} \in K_{k}} c_{\mathbf{i}}^{t} \leq c_{-}^{-q t} \sum_{\mathbf{i}^{\prime} \in Q_{k}} c_{\mathbf{i}^{\prime}}^{t} \leq 1
$$

this last inequality follows from repeated substitution of (3.9), using (3.4). Thus if $M^{s}(K)<1$, then for some $t<s$ we have $M^{t}(K) \leq \lim _{k \rightarrow \infty} \sum_{\mathbf{i} \in K_{k}} c_{\mathbf{i}}^{t} \leq c_{-}^{q t}$, so $M^{t}(K)<\infty$ and $\tau(s)<\tau(t) \leq 1$, since $\tau$ is strictly decreasing. It follows that (a) $\leq$ (b) and also that $M^{s}(K) \geq 1$ if $s$ is the number given by (b).

We now prove the results on dimensions; first we give the upper bound in the general case.

Proposition 3.3. Let $E$ be an s.s.s. set with respect to the similarities $\left\{S_{1}, \ldots, S_{k}\right\}$. Let $s$ be the number satisfying $\tau(s)=1$. Then

$$
\operatorname{dim}_{H} E \leq \underline{\operatorname{dim}}_{B} E \leq \overline{\operatorname{dim}}_{B} E \leq s .
$$


Moreover, if $M^{s}(K)<\infty$ (which will be the case if $\lim _{k \rightarrow \infty} \sum_{\mathbf{i} \in K_{k}} c_{\mathbf{i}}^{s}<\infty$ ), then $H^{s}(E)<\infty$.

Proof. Let $B$ be a closed ball such that $S_{i}(B) \subseteq B$ for $i=1, \ldots, m$. Let $\delta$ satisfy $0<\delta \leq|B|$. For all $\mathbf{i}=\left(i_{1}, i_{2}, \ldots\right) \in K$ we may find $k \in$ $\{0,1,2, \ldots\}$ such that

$$
c_{-} \delta<\left|S_{i_{1}} \circ \cdots \circ S_{i_{k}}(B)\right|=c_{i_{1}} c_{i_{2}} \cdots c_{i_{k}}|B| \leq \delta .
$$

Hence, writing $Q_{\delta}=\left\{\mathbf{i} \in K: c_{-} \delta<c_{\mathrm{i}}|B|=S_{\mathrm{i}}(B) \leq \delta\right\}$ we have that $E \subseteq$ $\bigcup_{\mathrm{i} \in Q_{\delta}} S_{\mathrm{i}}(B)$ is a cover of $E$ by sets of diameter at most $\delta$. If $N_{\delta}$ is the number of sets in this cover, then for $t>s$

$$
N_{\delta} \delta^{t}=\sum_{\mathbf{i} \in Q_{\delta}} \delta^{t} \leq c_{-}^{-t}|B|^{t} \sum_{\mathbf{i} \in Q_{\delta}} c_{\mathbf{i}}^{t} \leq c_{-}^{-t}|B|^{t} \sum_{k=1}^{\infty} \sum_{\mathbf{i} \in K_{k}} c_{\mathbf{i}}^{t} \leq M
$$

where $M<\infty$ is independent of $\delta$, using Proposition 3.2(c). It follows that $\overline{\operatorname{dim}}_{B} \leq t$ for all $t>s$, and so $\overline{\operatorname{dim}}_{B} E \leq t$.

Clearly, if $K \subseteq \bigcup_{\mathbf{i} \in Q} I_{\mathbf{i}}$, then $E \subseteq \bigcup_{\mathbf{i} \in Q} S_{\mathbf{i}}(B)$, so $H_{\delta}^{s}(E) \leq|B|^{s} M_{k}^{s}(E)$ if $\delta \geq c_{+}^{k}$, and letting $k \rightarrow \infty$ gives that $H^{s}(E) \leq|B|^{s} M^{s}(K)$.

To obtain a lower bound, we require the open set condition (3.2) to hold. In order to utilise this, we require the following geometrical lemma.

Lemma 3.4. Let $\left\{V_{i}\right\}$ be a collection of disjoint open subsets of $\mathbf{R}^{n}$ such that each $V_{i}$ contains a ball of radius $a_{1} r$ and is contained in a ball of radius $a_{2} r$. Then any set $U$ of diameter at most $r$ intersects at most $b_{1} \equiv\left(1+2 a_{2}\right)^{n} a_{1}^{-n}$ of the closures $\left\{\bar{V}_{i}\right\}$.

Proof. This is [7, Lemma 9.2].

Theorem 3.5. Let $E$ be s.s.s. with respect to a family of similarities $\left\{S_{1}, \ldots, S_{k}\right\}$ which satisfy the open set condition (3.2), and let $s$ be the number satisfying $\tau(s)=1$. Then $H^{s}(E)>0$ and

$$
s=\operatorname{dim}_{H} E=\underline{\operatorname{dim}}_{B} E=\overline{\operatorname{dim}}_{B} E .
$$

Proof. In view of Proposition 3.3, it is enough to show that $H^{s}(E)>0$. By Proposition 3.2 $M^{s}(K)>0$, so by Proposition 3.1 there is a compact subset $A_{0}$ of $K$ such that the Borel measure $\mu$ defined by $\mu(W)=M^{s}\left(A_{0} \cap W\right)$ for $W \subseteq J$ is supported by $K$ and satisfies $\mu(K)>0$ and

$$
\mu\left(I_{\mathbf{i}}\right) \leq b c_{\mathbf{i}}^{S}
$$

for all $\mathbf{i} \in K_{k}$ for all $k$. We use the mapping $a: K \rightarrow \mathbf{R}^{n}$ given by (2.2) to pull back this measure to $\mathbf{R}^{n}$. Letting

$$
\tilde{\mu}(U)=\mu\{\mathbf{j}: a(\mathbf{j}) \in U\}
$$

for $U \subseteq \mathbf{R}^{n}$ defines a Borel measure supported by $a(K)=E$. Let $V$ be an open set satisfying (3.2), and let $U \subseteq \mathbf{R}^{n}$ satisfy $0<|U| \leq|V|$. Let $Q$ be the set of indices

$$
Q=\left\{\left(i_{1}, \ldots, i_{k}\right): c_{i_{1}} c_{i_{2}} \cdots c_{i_{k}}|V|<|U| \text { and } c_{i_{1}} c_{i_{2}} \cdots c_{i_{k-1}}|V| \geq|U|\right\} .
$$

Since $c_{-}|U| \leq\left|S_{\mathbf{i}}(V)\right|<|U|$ for $\mathbf{i} \in Q$, there are at most $b_{1}$ indices in the family $Q_{0}=\left\{\mathbf{i} \in Q: U \cap S_{\mathbf{i}}(\bar{V}) \neq \varnothing\right\}$, where $b_{1}$ is independent of $U$, using Lemma 3.4. (Note that the sets $\left\{S_{\mathbf{i}}(V): \mathbf{i} \in Q_{0}\right\}$ are disjoint since the open set 
condition holds.) Thus if $a(\mathbf{j}) \in U$ then $\mathbf{j} \mid k \in Q$ for some $k$, so $\mathbf{j} \in I_{\mathbf{i}}$ for some $i \in Q_{0}$. From (3.11)

$$
\tilde{\mu}(U)=\sum_{\mathbf{i} \in Q_{0}} \mu\left\{\mathbf{j} \in I_{\mathbf{i}}\right\} \leq b \sum_{\mathbf{i} \in Q_{0}} c_{\mathbf{i}}^{s} \leq b b_{1}|V|^{-s}|U|^{s} .
$$

Since $\tilde{\mu}$ is supported by $E$, the mass distribution principle [6, Proposition 4.2] implies that $H^{s}(E)>0$.

We end with an open question. It is known [6] that if $E$ is a self-similar set, then $\operatorname{dim}_{H} E=\underline{\operatorname{dim}}_{B} E=\overline{\operatorname{dim}}_{B} E$ regardless of whether or not the open set condition holds. Whilst it seems very unlikely that this is the case for general sub-self-similar sets without the open set condition, I have been unable to find a counterexample.

\section{REFERENCES}

1. C. Bandt and S. Graf, Self-similar sets 7. A characterization of self-similar fractals with positive Hausdorff measure, Proc. Amer. Math. Soc. 114 (1992), 995-1001.

2. C. Bandt, Self-similar sets I. Topological Markov chains and mixed self-similar sets, Math. Nachr. 142 (1989), 107-123.

3. G. A. Edgar, Measure, topology and fractal geometry, Springer-Verlag, 1990.

4. K. J. Falconer, The geometry of fractal sets, Cambridge Univ. Press, 1985.

5. _ The Hausdorff dimension of self-affine fractals, Math. Proc. Cambridge Phil. Soc. 103 (1989), 339-350.

6. _ Dimensions and measures of quasi-self-similar sets, Proc. Amer. Math. Soc. 106 (1989), 543-554.

7. _ Fractal geometry-Mathematical foundations and applications, Wiley, 1990.

8. J. E. Hutchinson, Fractals and self-similarity, Indiana Univ. Math. J. 30 (1981), 713-747.

9. P. Mattila, Geometry of sets and measures in Euclidean spaces, Cambridge Univ. Press, 1995.

10. R. D. Mauldin and S. C. Williams, Hausdorff dimensions in graph directed constructions, Trans. Amer. Math. Soc. 309 (1988), 811-829.

11. C. A. Rogers, Hausdorff measures, Cambridge Univ. Press, 1970.

12. D. W. Spear, Measure and self-similarity, Adv. Math. 91 (1992), 143-157.

Mathematical Institute, University of St. Andrews, North Haugh, St. Andrews, Fife KY16 9SS, UK 